\section{Children Australia}

www.cambridge.org/cha

\section{Editorial}

Cite this article: Ainsworth F, Sanders R, and Lehmann J (2020). Poverty and child abuse and neglect. Children Australia 45: 199-201. https:// doi.org/10.1017/cha.2020.62

Author for correspondence:

Rachael Sanders,

Email: r.sanders@latrobe.edu.au

\title{
Poverty and child abuse and neglect
}

\section{Frank Ainsworth ${ }^{1}$, Jennifer Lehmann ${ }^{2}$ and Rachael Sanders ${ }^{3}$}

${ }^{1}$ Senior Principal Research Fellow (Adjunct), Department of Social Work and Human Services, James Cook University, Townsville Campus, Queensland, Australia, 4811; ${ }^{2}$ Adjunct Senior Lecturer, La Trobe Rural Health School, La Trobe University, Edwards Road, Bendigo, Australia, 3550 and ${ }^{3}$ Lecturer, La Trobe Rural Health School, La Trobe University, Edwards Road, Bendigo, Australia, 3550

The relationship between poverty and child abuse and neglect (CAN) came to the fore in 2016 following a publication of a Rowntree Foundation-funded report authored by Bywaters and colleagues in the United Kingdom. The Rowntree Foundation continues to pursue this topic with a weekly posting on their website (http://www.jrf.org.uk). Poverty and CAN has also received significant attention in the USA. Recently, the Anne E. Casey Foundation announced a 'first-of-its kind partnership' between the US Children's Bureau, Casey Family Programs and Prevent Child Abuse America (http://www.aecf.org). This partnership aims to redesign child welfare into child and family well-being systems, which certainly means addressing parental poverty and revising income support programmes.

Currently, there is a paucity of attention on this issue in Australia. There is no philanthropic foundation activity like that of Rowntree or the Anne E. Casey Foundation that focuses on poverty and CAN, hence this Special Issue (Part A) in Children Australia. Three of the papers in this section of the journal are from Australia with two further papers from Italy and Zimbabwe. The three Australian papers are from New South Wales (NSW), Western Australia (WA) and Queensland (QLD). Each paper has a different focus - social origins (Ainsworth), legal disadvantage (Harrison et al.) and service delivery (Bennett et al.) - but all tell a similar story about how parental poverty makes dealing with child protection authorities an impossible task. And, that certain aspects of child protection practice cause continuing harm to parents from whom children have been removed (Ainsworth \& Hansen, 2012).

While the Ainsworth paper gives an overview of the research on poverty and CAN in the UK and USA, the most powerful of the papers presented in the Special Issue papers is from the Family Inclusion Network (FIN) in Townsville (for a history of FIN, see Ainsworth \& Berger, 2014). This paper presents six case studies that report the lived experience of parents. These case studies highlight the imbalance of power between the state and parents involved with child protection services, and the way the state uses its power to further disenfranchise families. It is clear in this article that the way in which child protection services perform their duties can be characterised as an attack on the poor. For a country like Australia that talks about giving everybody a fair go, and where state and territory protection services claim to be working in partnership with parents, this is an obscenity. As Harrison et al. state in their paper, it represents a 'fundamental blemish on the principles of due process and fairness'.

Given that the three Australian papers are from different states, it is reasonable to conclude that parental poverty, and the difficulties that flow from poverty, are common to all Australian states and territories. Certainly, in my experience, the situations described by FIN in Queensland are fully replicated in NSW.

The other two papers, one from Africa and the other from Europe are sharply different. The contribution from Noel Muridzo and Victor Chikadzi in Zimbabwe cites adverse living conditions, rurality, child labour, migration and the underlying poverty as key factors that contribute to child sexual abuse (CSA). These factors are not likely to be cited in Australia when CSA is under discussion, although remoteness and Indigenous status are now acknowledged as contributing to the wider findings of CAN (AIHW, 2020, Figure 5.4). A particular value of this paper is the reference list, as it is a source of African materials about poverty, CSA and CAN that will be new to many readers of Children Australia, including myself.

The Italian paper by Maria Bezze and colleagues focuses on using cash transfers as a typical European approach to tackling child poverty. It is the only paper that reports on research studies into this issue. It also highlights how outcome evaluation can promote improved child protection practice. In that respect, Fondazione Emanuela Zancan is leading the way in the Italian efforts to address the issue of poverty and CAN.

Thanks are due to all the authors who responded to the call for papers for this edition. Hopefully, across time, more attention will be given as to how poverty and CAN are related. Not to do so would be to support injustice.

The second part of this issue contains all remaining articles. Because this is the final issue of Children Australia, we have 'cleared the books' and this issue of the journal has a higher than usual volume of papers. As such, there is an interesting mix of topics and types of papers, with various forms of reviews being particularly prevalent. The first paper, however, is a practice 
commentary by me about practitioners' rapid transition to telehealth in response to the need for social distancing during COVID-19 times. Numerous conversations with practitioners about how they coped with quickly shifting their practice online led me to believe that sharing their experiences and insights might be useful for sole practitioners who reported feeling quite isolated and unsure during the process of change. After having interviewed a number of practitioners, I put together this commentary of shared experiences and ideas that might be useful for practitioners now and into the future, should a similar event occur.

The first of the six reviews found in this issue is by Jade Purtell, Philip Mendes and Bernadette Saunders who have used the extant literature to examine care leavers who become parents, often within a short time of leaving care. In conducting their analysis, the authors test the relevance of Ambiguous Loss Theory in understanding how removal from families of origin and placement experiences may affect young people and lead to 'wanted' pregnancies. While knowledge of the disadvantage experienced by care leavers is familiar to those working in the sector, we know much less about how exploitation and coercion might play a role in their lives. The literature fails to investigate the short-, medium- and long-term impacts of removal from family on children and young people and, consequently, any ongoing effects of the ambiguous loss faced by young people removed from family members. The authors conclude there is a need for further research to focus on the role of ambiguous loss in explaining care leaver early parenting and the cycle of child protection involvement for care leavers unable to care for their children.

Stephan Lund and Cathryn Stokes undertook a brief scoping review of the educational outcomes for children in care in Australia. The main themes, drawn from 25 papers, included stigma and low expectations, school disruption and absenteeism, issues within the care and education systems and the importance of good relationships with supportive adults. The authors infer that young people in care often experience much worse educational outcomes than their peers, and recommend a restructuring of both the care and education systems; with an emphasis on building supportive relationships and prioritising education for these young people.

Building on the previous paper that calls for improved educative support for young people who have been abused or neglected, Simone Collier and her colleagues draw our attention to the integral position that the education system, and the workers found therein, have to the protection and education of these children; many of whom have experienced and suffer the lasting consequences of developmental trauma. The authors examine the prevailing practice frameworks, models, approaches and programmes implemented in mainstream primary schools that aim to meet the learning needs of children who have experienced trauma in their early childhood years. The authors' interrogation of current frameworks highlight both the benefits of these agendas, but also where they fall short and what might be useful to help remedy the disadvantages experienced by these children in the education system.

It is very timely that a paper focussing on online counselling can be presented. The pandemic that continues to face the world has led to some distinctive changes to the way services are conducted and online counselling is one of these. Mary Jo McVeigh and Susan Heward-Belle have turned their attention to the ethical issues in relation to online counselling and this article presents the findings of a review of the literature on the topic. The results of their review highlight a number of common ethical issues discussed across the literature, but with a major gap being on the particular issues for children and young people. A continued privileging of the adult's voice over children and young people's needs was evident in the analysis and, with the focus on children and young people who have been abused, there continue to be some very real dilemmas for counselling staff using online platforms.

Specialising in the topic of filicide, Thea Brown, Danielle Tyson and Paula Fernandez Arias, have prepared an analysis of data that identifies the profiles of victims and perpetrators. They discuss the constellation of circumstances and stressors associated with each of the parental perpetrator groups, including the perpetrators' contact with, and mostly unsuccessful use of, services. Using two categories of research data - large-scale studies such as the 10-year plus retrospective studies of filicide deaths in NSW; and several smaller scale studies on specific areas of child death such as maternal infanticide - the analysis is used to answer three key questions. These are: (1) What is the incidence of filicide deaths in Australia and how does this incidence compare with other similar countries? (2) What are the characteristics of filicide victims and perpetrators in Australia and how do these compare with those from other countries? And (3) What are the implications for prevention that arise from the answers to these questions? Their exploration points to a number of complex factors including poverty, mental health and criminal history that will need to be addressed by services for this group of parents.

The final review in this issue is a systematic review by Owczarek and colleagues who explore teenagers' worries. Given excessive worry can impact on young people's developmental trajectory, it important to understand what contemporary teens worry about, so measures can be put in place to help them remove or navigate these in a way that lessens any negative impact. The authors discuss seven decades worth of adolescent worry, identifying a range of themes, with 'school performance' being the most consistent across studies. This investigation highlighted the notion that worries are influenced by culture, socio-economic status, political and other national events, gender norms, religiosity and so on, which makes the concept non-transferrable across regions and cohorts; ultimately making it difficult to develop a uniform way of measuring worry, let alone developing universal interventions.

The first two research articles presented here are by Renée O'Donnell and colleagues. The first is a scoping study, which prioritises the voice of young people when transitioning from out of home care. Exploring the challenges associated with the transition from out of home care and how best to overcome these challenges, the authors spoke with young people and the wider care team to inform better practice. They found that the main challenges were associated with inadequate processes, instability within the family unit, financial difficulties and a lack of independence during care, with two key solutions being improved life-skills training while in care and better after-care support.

The second article by Renée O'Donnell and others is an evaluation of the Aboriginal Cradle to Kinder (AC2K) programme delivered by the Victorian Aboriginal Child Care Agency (VACCA). Like the previous study, the authors wanted to highlight and prioritise service users' perspectives, as well as those of the workers who deliver the programme. The aim was to identify the strengths, limitations and recommendations of the AC2K. The programme was well received and thought to promote greater connection with culture and improved parenting skills; however, they identified caseload difficulties and recommendations were 
made for improved connections with child protection as a way of enhancing the programme further.

The second evaluation, this time of a residential education programme for disengaged young people by Paul Mastronardi, Frank Ainsworth and Jonathan Huefner, found that the Boystown model of promoting improved educational outcomes for young people who are disengaged from school to be effective in progressing their education and reducing behavioural issues related to school refusal. A key message in this paper is the importance and relevance of collecting and using outcome data in agencies to inform practice and increase programme effectiveness.

Sanders, Lehmann and Gardner report on new parents' experiences of using the available range of parenting resources that help guide parenting. The resources used by new parents include professional services, peers, family and friends, and written material. Expert opinion was valued when problems occurred, but frustration was felt by parents when faced with mixed messages. Many parents did not want overt and unsolicited advice, but often relied on informal advice and support from peers.

The final paper for not only this issue, but for the journal as a whole, represents what this journal tried to achieve over its 45 -year existence - not only to disseminate knowledge and ideas, but to shine a spotlight on social inequalities and systems that could work better to support children, young people and families in reaching a true sense of well-being. The final paper, by McCausland and Leanne Dowse, does this in a most compelling and troubling way by stepping the reader through the life of Casey, a young Indigenous girl/woman with a cognitive disability whose early life experience perhaps encapsulates the failings of our systems to support some of the most vulnerable members of our society. I imagine she experienced moments of kindness, generosity, compassion and being 'held', but overall, her story appears to be one of trauma, negligence and inadequate support and care. The authors use an interesting mixed-methods methodology to track Casey's engagement with service systems as a way of highlighting the way that people with a cognitive disability are 'managed' by the health, housing, disability, human services, police, legal, court and justice agencies, often in uncoordinated and inefficient ways. The paper highlights the ways that people with complex needs often find themselves being 'managed' by the justice system because of a lack of appropriate early intervention and community-based options, particularly in remote areas. The authors promote alternative community-led ways of supporting them that not only better meet their human rights but might also use the community purse in more appropriate and ethical ways.

Finally, this issue concludes with two book reviews by Frank Ainsworth. Frank has been a staunch advocate for the rights of children and parents in the justice system, and social policy more broadly, and has reviewed many books for Children Australia on these topics over the years, so we consider it only fitting that the journal closes with one of Frank's reviews.

Again, we thank all of you who contributed to the journal in one form or another over its 45-year life. It has been a pleasure to work with you on this journal for some of that time.

\section{References}

Ainsworth, F., \& Hansen, P. (2012). Doing harm while doing good: The child protection paradox. Child and Youth Services, 33(2), 146-157.

Ainsworth, F., \& Berger, J. (2014). Family inclusive child protection practice: The history of the Family Inclusion Network and beyond. Children Australia, 39(2), 60-64.

Anne E. Casey Foundation (2020). First-of-its kind partnership aims to redesign child welfare into child and family well-being systems. Baltimore. http://www. aecf.org

Australian Institute of Health and Welfare (2020). Child protection Australia 2018-19. https://www.aihw.gov.au/getmedia/3a25c195-e30a-4f10-a052adbfd56d6d45/aihw-cws-74.pdf.aspx?inline=true

Bywaters, P., Bunting, L., Davidson, G., Hanratty, J., Mason, W., McCartan, C., \& Steils, N. (2016). The relationship between poverty, child abuse and neglect: An evidence review. Rowntree Foundation. 